\title{
Quality of veiled olive oil: Role of turbidity components
}

\author{
Carlotta Breschi, Lorenzo Guerrini*; Ferdinando Corti, Luca Calamai, Paola Domizio, Alessandro Parenti, and \\ Bruno Zanoni
}

Department of Agriculture Food, Environment and Forestry (DAGRI), Università Degli Studi di Firenze, 50121 Florence, Italy

*Corresponding Author: Lorenzo Guerrini, Department of Agriculture Food, Environment and Forestry (DAGRI), Università Degli Studi di Firenze, 50121 Florence, Italy. Email: lorenzo.guerrini@unifi.it

Received: 28 May 2021; Accepted: 2 September 2021; Published: 18 September 2021

(c) 2021 Codon Publications

OPEN ACCESS (C) (1) (9)(2)

PAPER

\begin{abstract}
This study investigated the effects of water and content of solid particles, taken together as well as separately, on stability of veiled olive oil. The following oil samples were obtained through four different separation treatments: veiled, filtered, 'solid-only', and 'water-only'. Changes in chemical, microbial, and sensory characteristics were evaluated during storage (240 days). A significant effect of hydrolysis was shown in veiled and 'water only' oils; in 'solid-only' oils, a slow increase of phenols was observed. A notable microbial activity, with resulting formation of volatile metabolites and sensory defects, was observed in veiled samples. Filtered oils underwent less significant changes.
\end{abstract}

Keywords: biophenols, hydrolysis, oil-borne microorganism, olive oil quality, volatile compounds

\section{Introduction}

Preservation of quality during storage is an important subject for extra-virgin olive oil (EVOO) producers (International Olive Council [IOC], 2018). Good preservation practices are essential to maintain quality of EVOOs up to shelf-life. Moreover, sensory profile and contents of phenolic compounds change during storage, leading to a decrease in hedonic and health characteristics. Filtration is one of the most used stabilization processes for EVOOs (Guerrini et al., 2015). Interest in unfiltered oils has increased during last few years (Bimbo et al., 2020).

Cloudy aspect of veiled extra-virgin olive oils (VEVOO) is due to the presence of micro-droplets of water and fragments of olive pulp and stone suspended/dispersed in the oil phase (Lercker et al., 1994; Koidis et al., 2008). Furthermore, different combinations of water and insoluble solids can lead to different 'turbidities' in VEVOOs (Breschi et al., 2019). The same degree of turbidity of a
VEVOO could be characterized by different water contents, insoluble solid contents, water activity, and/or microbial contamination. Therefore, VEVOO turbidity is not a dichotomous variable, but it is a continuous variable of different proportions of water, insoluble solids, microbial contamination, and water activity (Breschi et al., 2019).

The difference between VEVOO and filtered extravirgin olive oil (FEVOO) during storage is still a controversial and widely studied topic for the quality of olive oil (Cayuela-Sànchez and Caballero-Guerrero, 2019). Some authors have proclaimed that suspended particles play a stabilizing function during storage because most phenolic compounds present in olive oil, having hydrophilic nature, are located in water droplets and insoluble solids (Lonzano-Sànchez et al., 2010). Therefore, the presence of suspended particles acts as an antioxidant, providing greater oxidative stability (Lercker et al., 1994; Ambrosone et al., 2002; Koidis and Boskou, 2006; Migliorini et al., 2009). Moreover, the suspended 
particles act as buffer against increase in free fatty acid (FFA) and hydrolytic degradation (Frega et al., 1999).

On the other hand, in literature, improvement in shelf life because of elimination of sediment by filtration was evidenced. In VEVOO, solid particles and water micro-droplets trap microorganism, mainly yeasts, and constitute a perfect environment for microbial survival (Guerrini et al., 2015; Ciafardini and Zullo, 2002a; Ciafardini and Zullo, 2002b; Zullo and Ciafardini, 2020a; Zullo and Ciafardini, 2020b). In veiled oils (VOs), microbial metabolism promoted by a water activity of more than 0.6 (Breschi et al., 2019; Bubola et al., 2017) was responsible for fast behavior of sensory defects, such as 'fusty' and 'muddy-humidity', and oil debittering phenomena (Zullo and Ciafardini, 2020b ; Zullo et al., 2013; Zanoni, 2014; Cayuela et al., 2015; Guerrini et al., 2020a; Zullo et al., 2020). Moreover, the yeast present in VEVOO was responsible for oxidation of phenolic compound and hydrolysis of triacylglycerol (Zullo et al., 2013; Romo-Sánchez et al., 2010; El Haouhay et al., 2018; Ciafardini and Zullo, 2018). Water content also affects the hydrolytic activity of olive oil; hydrolysis is faster at the interface between the two phases of oil and water (Xenakis et al., 2010). This effect has been demonstrated with a higher increase of hydroxytyrosol and tyrosol in veiled olive oils than in filtered oils (FO) (Brenes et al., 2001; Fregapane et al., 2006; Fortini et al., 2016; Guerrini et al., 2020b).

Given the conflicting results about the role of turbidity on the stability of VEVOOs, in this work, an original research was carried out on the different role of water and insoluble solid particles content during storage of EVOO by testing a wide spectrum of olive oil 'turbidities'.

The present work is a part of wide study on the turbidity and stabilization of olive oil. The first contribution (Breschi et al., 2019) allowed defining a set of analyses useful to study turbidities of olive oil based on its physical-chemical and microbiological characterization. Then a specific research (Guerrini et al., 2020b) was carried out on the role of water and microorganisms, the two factors that mostly compromise the stability of VEVOOs. Then the dynamics of development of 'fusty' sensory defect and the hydrolysis of phenolic compounds were studied (Guerrini et al., 2020a), since these phenomena were always present in the analyzed VEVOOs, with the aim of establishing an adequate filtration schedule.

Finally, the present work aimed (i) to study the contribution of dispersed water droplets or solid particles, which, to different extent, contribute to turbidity in VEVOOs and affect the qualitative characteristics of olive oil during a simulated medium storage period, and (ii) how important the qualification of olive oil turbidity could be to plan separation techniques during crop seasons and storage of olive oil.

\section{Materials and Methods}

\section{Olive oil samples}

EVOO samples were extracted in October-November 2017 in an industrial continuous plant (TEM, Florence, Italy) in Azienda Agricola La Ranocchiaia (Florence, Italy). The plant was equipped with the following: olive cleaner, blade cutter crusher, sealed vertical malaxer (300 $\mathrm{kg}$ ), and two-phase horizontal centrifuge (i.e., decanter). The malaxation was carried out at $18^{\circ} \mathrm{C}$ for $20 \mathrm{~min}$.

Six different 300-kg batches of blend of olive cultivars, harvested in Tuscany, were processed on three different days in 2017: olive oils \#1 and \#2 were processed on October 31, 2017; olive oils \#3 and \#4 were processed on November 7, 2017; and olive oils \#5 and \#6 were processed on November 28, 2017.

Six 20-kg batches of oil from each batch of blended olive cultivars were collected at the end of 'decanter', immediately transferred to the laboratory, and subjected to the following four different water and solid particle separation treatments: (1) first $1 \frac{1}{4}$ of oil batches ( $5 \mathrm{~kg}$ of oil) were untreated, forming $\mathrm{VO}$ samples for this study (i.e., samples VO\#1-VO\#6); (2) second $1 / 4$ of oil batches (5 kg of oil) were filtered using a portable filter press (Colombo Inox 12, Rover Pompe, Padua, Italy) equipped with five filter sheets (Rover 8, 3- $\mu$ m cut-off, Rover Pompe, Padua, Italy), forming $\mathrm{FO}$ samples for this study (i.e., samples FO\#1-FO\#6); (3) third $1 / 4$ of oil samples (5 $\mathrm{kg}$ of oil) were freeze-dried (Modulyo, Edwards, Milan, Italy), forming the 'solid particle-only' (SO) samples for this study, that is, freshly extracted olive oil containing solid particles only without water (i.e., samples SO\#1-SO\#6); and (4) last $1 / 4$ of oil samples ( $5 \mathrm{~kg}$ of oil) were filtered with glass wool using a filter aid to separate solid particles, forming 'water-only' (WO) samples for this study, that is, freshly extracted olive oil containing water only without solid particles (i.e., samples WO\#1-WO\#6).

All oil samples obtained (4 treatments $\times 6$ different oil batches $=24$ oil samples) were bottled in 0.25 - $\mathrm{L}$ clear glass bottles with a headspace of about $8 \%$ of bottle's volume, and immediately analyzed to measure turbidity characterization parameters (i.e., degree of turbidity, water content, water activity, solid particles content, and microbial cell count) as described in Breschi et al. (2019). Chemical characteristics (FFA, peroxide value [PV], ultraviolet [UV] spectroscopic indices [K232, K270, and $\Delta \mathrm{K}]$, and content of phenolic and volatile compounds) and sensory attributes were also measured. 
For storage test, all olive oil samples ( 4 treatments $\times 6$ different oil batches $\times 4$ storage periods $=96$ oil samples) were bottled in 0.25 - L clear glass bottles with a headspace of about $8 \%$ of bottle's volume. These were stored at room temperature $\left(20^{\circ} \mathrm{C}\right)$ in a chamber $(1.3 \times 1.0 \times 0.8 \mathrm{~m})$ with internal walls covered with reflective material and a light intensity of 1,900 lux (Master TL-D 90 Graphica lamp, 35 W/390, Philips, Amsterdam, the Netherlands) for $12 \mathrm{~h}$ per day. After 45, 120, 180, and 240 days of storage, the olive oil samples were analyzed to measure FFA, $\mathrm{PV}, \mathrm{K} 232, \mathrm{~K} 270, \Delta \mathrm{K}$, and phenolic and volatile compounds content and sensory parameters.

\section{Analyses}

Turbidity characterization parameters and microbial cell count The degree of turbidity was measured in nephelometric turbidity unit (NTU) using a Hach Model 2100 turbidimeter (Hach, Loveland, CO, USA). Water content, calculated as percent of water content weight/100-g olive oil sample (\% w/w), was analyzed with a Karl Fischer Kit for visual water determination without titrator (37858 HYDRANAL - Moisture Test Kit, Honeywell Fluka, Bucharest, Romania). Water activity (Aw) was measured using a Rotronic Hygroskop DT hygrometer (Michell Italia Srl, Milan, Italy). The solid particles content, calculated as the difference in weight and quantified as percentage of solid particles weight/100-g olive oil sample (\% w/w), was measured using the method described in literature (Zullo and Ciafardini, 2018), and calculated by weighing the difference and quantified as $\% \mathrm{w} / \mathrm{w}$. Microorganisms were enumerated according to the method reported in literature (ZULLO et al., 2010): an aliquot of each sample (i.e., $\approx 20 \mathrm{~mL}$ ) was taken from each bottle under sterile conditions and filtered through a $0.45-\mu \mathrm{m}$ sterile nitrocellulose membrane. Then the filtered content was transferred into a 50-mL sterile Falcon tube containing $20-\mathrm{mL}$ sterile physiological solution $(0.85 \% \mathrm{NaCl})$ and homogenized using UltraTurrax (mod. T25 homogenizer, IKA Milan, Italy). Of each homogenized sample, $200-\mu \mathrm{L}$ serial dilution was placed on YPD agar medium. Colonies were counted after $48-72 \mathrm{~h}$ of incubation at $28^{\circ} \mathrm{C}$.

\section{Chemical and sensory parameters}

The FFA (\% oleic acid), PV (meq $\mathrm{O}_{2} \mathrm{~kg}^{-1}$ ), and UV spectroscopic indices (K232, K270, and $\Delta \mathrm{K})$ were measured according to the official EU method (REG. 2016/2095). Extraction, identification, and determination of phenolic compounds was performed in agreement with the official IOC method (IOC/T.20/Doc.29/Rev.1; International Olive Council [IOC], 2017) using an HPLC apparatus comprising Agilent 1200 series system (Agilent technologies, Santa Clara, CA, USA). The system was composed of a quaternary pump equipped with a diode-array detector and autosampler. The analytical conditions were as follows: HPLC column: LiChroCART ${ }^{\circ}$ 250-4.6 Purospher STAR RP-18E, $5 \mu \mathrm{m}(250 \times 4.6$-mm id; Merck KGaA $)$ equipped with a LiChroCART ${ }^{\circ}$ 4-4 Purospher STAR $\mathrm{RP}-18 \mathrm{E}, 5-\mu \mathrm{m}$ pre-column $(4 \times 4 \mathrm{~mm})$. Contents of phenolic compounds in oil samples were studied as total content, content of polyphenols from different family groups (sum of oleuropein and its derivates, sum of ligstroside and its derivates, phenolic acids, flavonoids, and lignans), and content of single representative compounds in EVOO (hydroxytyrosol and tyrosol). Moreover, R-index, which relates the content of the more hydrolysed phenols (hydroxytyrosol and tyrosol) to the less hydrolysed ones (oleuropein and its derivates and ligstroside and its derivates) was calculated as follows (Fiorini et al., 2018):

$$
\mathrm{R} \text {-index }=\frac{\text { hydroxytyrosol }+ \text { tyrosol }}{\text { oleuropein and its derivates }+}
$$

The content of volatile organic compounds in olive oil was determined using the combination of headspace solid phase microextraction (HS-SPME) and gas chromatography-mass spectrometry (GC-MS) technique as described in literature (Fortini et al., 2017). Analyses were carried out by weighing $4.3 \mathrm{~g}$ of sample and $0.1 \mathrm{~g}$ of internal standard mixture (ISTD MIX) in 20-mL screwcap vials fitted with a PTFE/silicone septum. After 5 min of equilibrium at $60^{\circ} \mathrm{C}$, the SPME fiber $(50 / 30 \mu \mathrm{m} \mathrm{DVB} /$ CAR/PDMS by Supelco, Darmstadt, Germany) was visible in the vial headspace for 20 min while being subjected to orbital shaking $(500 \mathrm{rpm})$. Then the fiber immediately desorbed for $2 \mathrm{~min}$ in a gas chromatograph injection port operating in split less mode at $260^{\circ} \mathrm{C}$. The identification of volatile compounds was performed by gas chromatography coupled with quadrupole mass spectrometry using a GC-MS scientific trace system (Thermo Fisher, Waltham, MA, USA) equipped with a $30 \mathrm{~m} \times 0.25 \mathrm{~mm} \mathrm{ID} \mathrm{and}$ $0.25-\mu \mathrm{m}$ DF ZB-FFAP capillary column (Phenomenex, Torrance, CA, USA). The mass detector was operated in scan mode within a mass range of 30-330 Thomson (Th) at 1,500 Th/s, with an ionization energy (IE) of 70 eV. Compounds were identified and quantified $(\mathrm{mg} / \mathrm{kg}$ ) by comparing their mass spectra and retention period with those of ISTD MIX. These consisted of the following 11 compounds: 3,4-dimethyl phenol, 4-methyl2-pentanol, hexanoic acid-d11, 1-butanol-d10, ethyl acetate- $\mathrm{d} 8$, toluene- $\mathrm{d} 8$, ethyl hexanoate-d11, acetic acid-2,2,2-d3, 6-chloro-2-hexanone, 3-octanone, trimethylacetaldehyde.

The panel test was carried out according to the official IOC method (IOC/T.20/Doc.15/Rev.10; International Olive Council [IOC], 2018b). Three women and five men, aged 29-58 years, comprised the panel. All panelists were 
trained following the official IOC procedure (IOC/T.20/ Doc.14/Rev.5; International Olive Council [IOC], 2018a). The panelists worked for the Taste Commission of the Ministerodelle Politiche Agricole Alimentari, Forestali e del Turismo (MIPAAAFT-Italian Ministry of Agri-Food and Forestry Policy and Tourism). For the safety of panelists, WO samples, filtered on glass wool, were not tasted but only smelt out.

\section{Data processing}

A linear model that included two tested variables (treatment and storage period) and their interactions were used to fit the experimental data. Data were analyzed with Matlab R2017B software (MathWorks, Natick, MA, USA). A two-way mixed effect ANOVA was performed to assess significant differences $(p \leq 0.05)$. Treatment was considered a fixed effect variable, while storage period was taken as a random effect variable.

Six olive oil samples for each treatment were used as replicated for storage study. This choice was done to understand both the behavior of unfiltered oils related to filtered oils, regardless of individual oil turbidity characteristics, and the separated role of water and solid particles during storage of unfiltered olive oils.

\section{Results}

\section{Turbidity characterization}

Immediately after production, the six VEVOO samples (VO\#1-VO\#6) used in this study were characterized for different 'turbidities' (Breschi et al., 2019). The turbidity grade ranged between 800 and 1,700 NTU, with water content between 0.15 and $0.40 \% \mathrm{w} / \mathrm{w}$, water activity between 0.60 and 0.85 , and insoluble solids content between 0.10 and $0.45 \% \mathrm{w} / \mathrm{w}$. Microbial cell count was between 2.5 and $4.5 \log \mathrm{CFU} \mathrm{g}^{-1}$.

After treatments, turbidity characteristics of olive oil samples changed radically. FEVOO samples (FO\#1FO\#6) were characterized by a degree of turbidity grade (10-20 NTU), water $(0.04-0.05 \% \mathrm{w} / \mathrm{w})$, and insoluble solids content $(0.00 \% \mathrm{w} / \mathrm{w})$, water activity $(0.30-0.45)$, and microbial cell count $\left(0.00 \log C F U g^{-1}\right)$, which were statistically $(p>0.05)$ lower than VO samples. The WO olive oil (WO\#1-WO\#6) and SO olive oil (SO\#1-SO\#6) samples were characterized by turbidity characteristics, which were between VEVOO and FEVOO samples. The degree of turbidity grade for WO olive oil samples was between 40 and 90 NTU and that for SO olive oil samples between 150 and 240 NTU. These turbidity grades were characterized by different water content $(0.10-0.11 \%$ w/w for WO samples; and $0.02-0.04 \% \mathrm{w} / \mathrm{w}$ for SO samples), water activity value (0.45-0.75 for WO samples; and $0.30-0.40$ for SO samples), and insoluble solids content $(0.00 \% \mathrm{w} / \mathrm{w}$ for WO samples; and $0.15-0.40 \% \mathrm{w} / \mathrm{w}$ for SO samples). The microbial cell counts for $\mathrm{WO}$ and SO olive oil samples were 0.5-3.0 $\log \mathrm{CFU} \mathrm{g}^{-1}$ and 0.0-.7 $\log$ CFU g ${ }^{-1}$, respectively.

\section{Chemical parameters and microbial cell count}

All olive oil samples resulted from the values of chemical parameters, FFA, PV, K232, K270, and $\Delta \mathrm{K}$, in the 'extra-virgin' category during whole storage (Table 1). However, the spectroscopic indices (K232, K270, and $\Delta \mathrm{K}$ ) significantly increased during storage for all treatments $(p$ $\leq 0.01)$. VO samples had statistically higher FFA and $\Delta \mathrm{K}$ values than FO, SO, and WO samples. However, the highest value of K270 was determined in SO olive oil samples.

Microbial cell count was statistically significant for treatment. VO samples had a microbial cell count higher than FO samples; WO olive oil samples had a microbial cell count between VO and FO samples. SO olive oil samples had a microbial cell count between WO and FO samples (i.e., no significant difference than both WO and FO). No statistically significant variation occurred during storage time. However, interactions between time and treatment were statistically significant. In WO and SO olive oil samples, the microbial cell count decreased during storage, in FO samples it did not change, and in VO samples, the microbial contamination increased up to 120 days, then decreased (Figure 1).

\section{Content of phenolic compounds}

The content of phenolic compounds of oil samples was studied as total content, content of different family groups of polyphenols, and content of single representative compounds in EVOO, as described in literature (Breschi et al., 2019; El Riachy et al., 2011) (Table 2).

The total phenolic content was statistically significant ( $p \leq 0.001)$ for treatment. The content of total phenolic compounds was statistically higher in SO samples than in VO and WO samples, which had a higher content of total phenolic compounds than in FO samples (Table 2). The statistically significant higher content of total phenolic compounds in SO samples was also determined by the sum of oleuropein and its derivates and the sum of ligstroside and its derivates (Table 2). Instead, the content of hydroxytyrosol, tyrosol, and phenolic acids was statistically higher $(p \leq 0.001)$ in VO samples than in WO and SO samples, which had higher content of hydroxytyrosol, tyrosol, and phenolic acids than in FO samples (Table 2). 


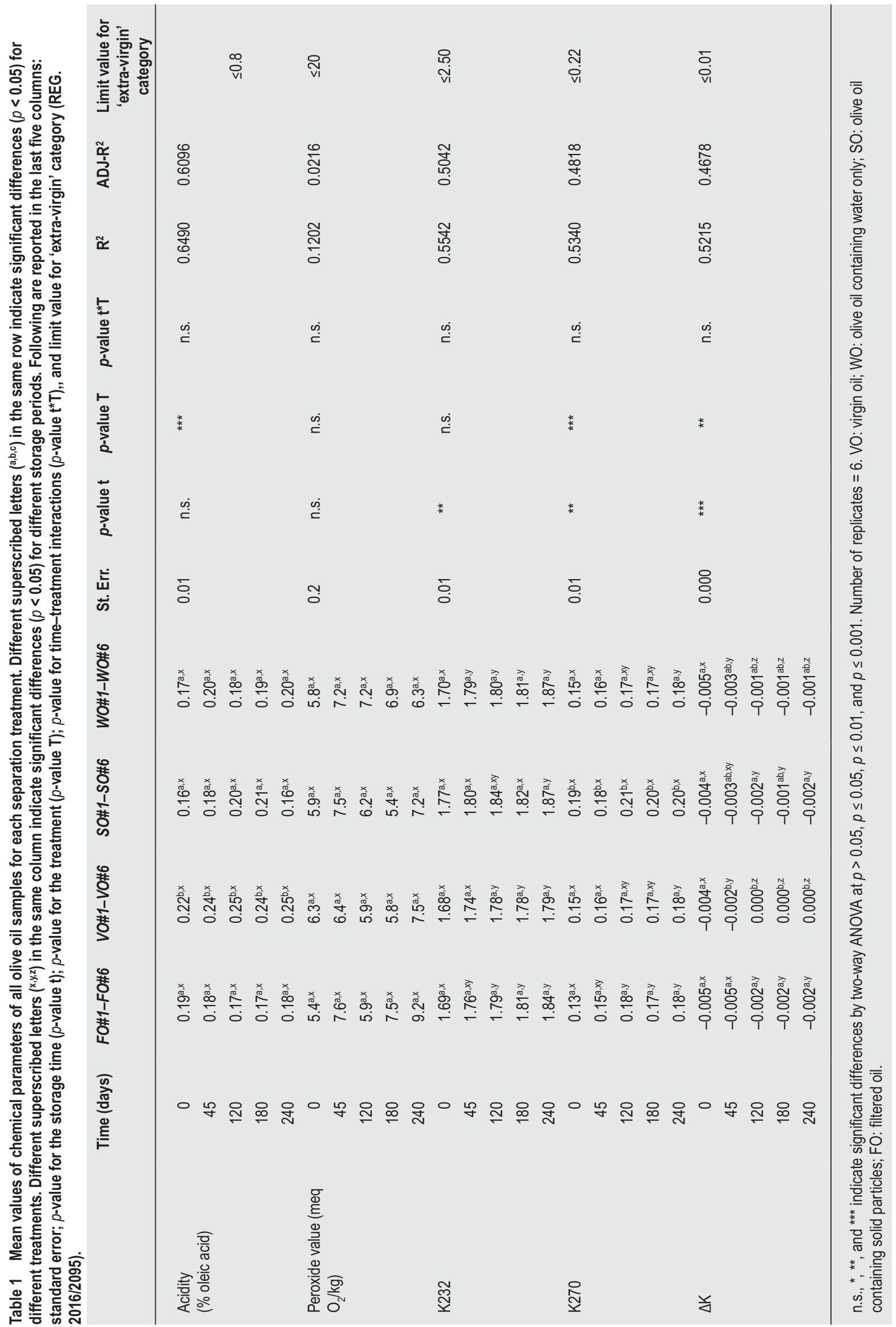




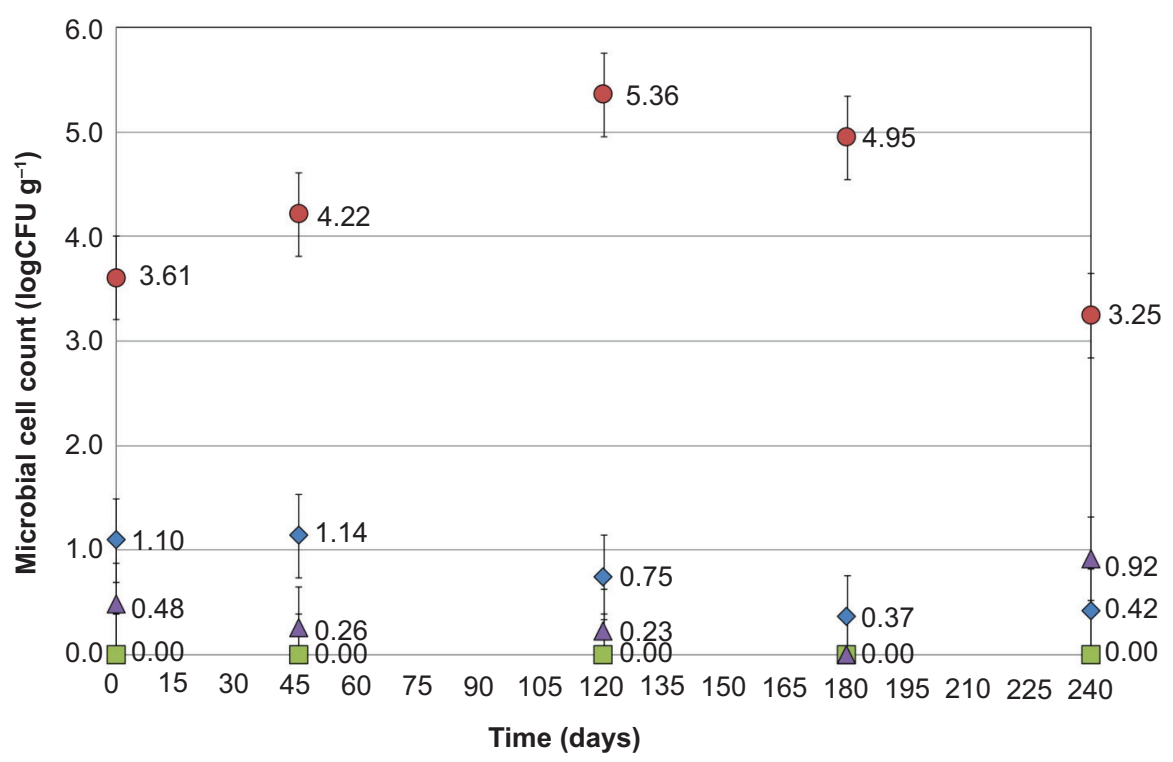

Figure 1 Mean contents and standard error of microbial cell count in samples of virgin oil (VO; red circle), olive oil containing water only (WO; blue diamond), olive oil containing solid particles (SO; purple triangle), and filtered oil (FO; green square) during storage. The $R^{2}$ and $A D J-R^{2}$ values of microbial cell count were 0.8522 and 0.8356 , respectively.

Significant interactions between storage period and treatment $(p \leq 0.001)$ were determined for hydroxytyrosol and tyrosol contents, which statistically increased faster in $\mathrm{VO}$ samples than in $\mathrm{WO}>\mathrm{SO}>\mathrm{FO}$ samples during storage (Table 2 ). Immediately after production (time $=0$ ), the content of both hydroxytyrosol and tyrosol was lower than $10 \mathrm{mg} \mathrm{kg}^{-1}$ and $5 \mathrm{mg} \mathrm{kg}^{-1}$, respectively, in all samples. During the 240 days of storage, the contents increased statistically in all samples except FO samples. VO samples had content of hydroxytyrosol and tyrosol statistically $(p \leq 0.001)$ higher than in FO samples. Content of both hydroxytyrosol and tyrosol in WO and SO samples was statistically different $(p \leq 0.001)$ and was between the content determined in $\mathrm{VO}$ and FO samples.

The contents of hydroxytyrosol, tyrosol, oleuropein, and ligstroside and their derivates were used to calculate R-index (R-index $=[$ hydroxytyrosol + tyrosol] $/[$ oleuropein and its derivates + ligstroside and its derivates]), a useful marker of the hydrolysis of secoiridoids (Fiorini et al., 2018). During storage R-index increased significantly $(p \leq 0.001)$ in all treatments, demonstrating degradation of phenols (Figure 2). The difference between treatments was statistically significant $(p \leq 0.001)$; except at the beginning of storage, the R-index of $\mathrm{VO}$ samples was always higher than that of FO and SO samples. WO samples had intermediate value of R-index. Moreover, timetreatment interactions were also statistically significant: in VO samples, the R-index gain was faster than in WO samples, which was faster than $\mathrm{SO}$ and $\mathrm{FO}$ samples.

The ratio of oxidized form of phenolic compounds to not oxidized form (OX:not OX) during storage period (Figure
3) was determined to observe the effect of oxidation on phenolic compounds. Immediately after production, FO samples showed the OX:not OX ratio value statistically $(p \leq 0.05)$ lower than in VO, WO, and $\mathrm{SO}$ samples. After 240 days of storage, increase in oxidized forms of phenolic compounds made a statistically significant difference in treatment: the OX:not OX ratio value was higher in FO and SO samples than in WO and VO samples.

\section{Content of volatile compounds}

The content of volatile compounds in olive oil samples was studied as described in literature (Guerrini et al., 2020a): pleasant lipoxygenase pathway (LOX pathway) volatile compounds with five (C5) and six (C6) carbon atoms; unpleasant volatile compounds related to 'fusty'/'mouldy'/'vinegary' defects; and unpleasant volatile compounds related to 'rancid' defect.

Some statistically significant differences $(p \leq 0.05)$ were identified in C5 and C6 branches of LOX pathway volatile compounds. A statistically significant main effect of filtration was detected in 1-hexanol, E-2-hexenol, Z-3hexenol, 1-penten-3-one, and E-2-penten-1-ol volatile compounds (Figure 4). The content of all these volatile compounds was higher in VO samples than in FO, WO, and $\mathrm{SO}$ samples.

The same statistically significant difference was also determined in 3-methyl-butanal, 2-octanol, and 2-nonanone unpleasant volatile compounds related to 'fusty' defect (Figure 5). Moreover, a statistically significant 


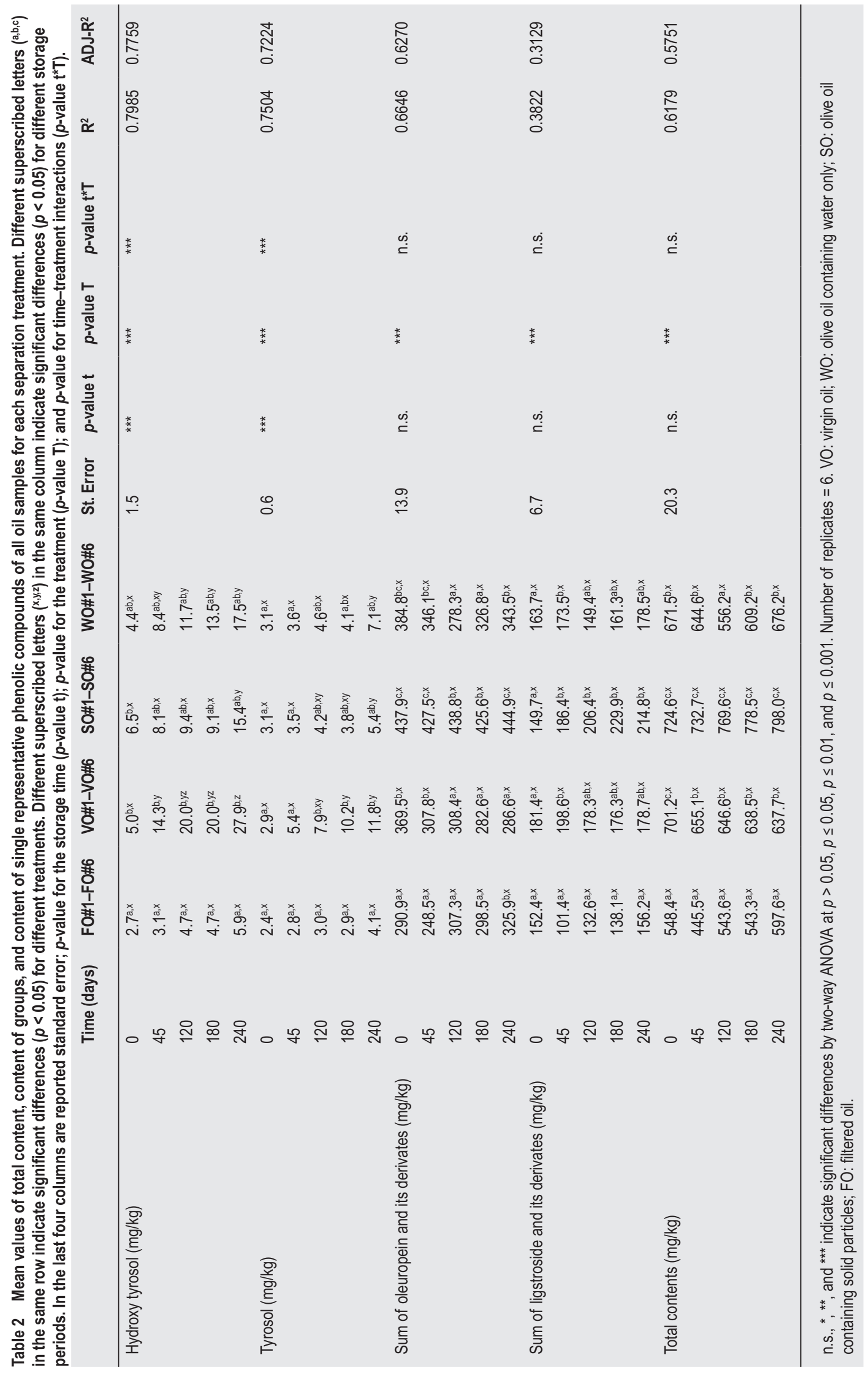




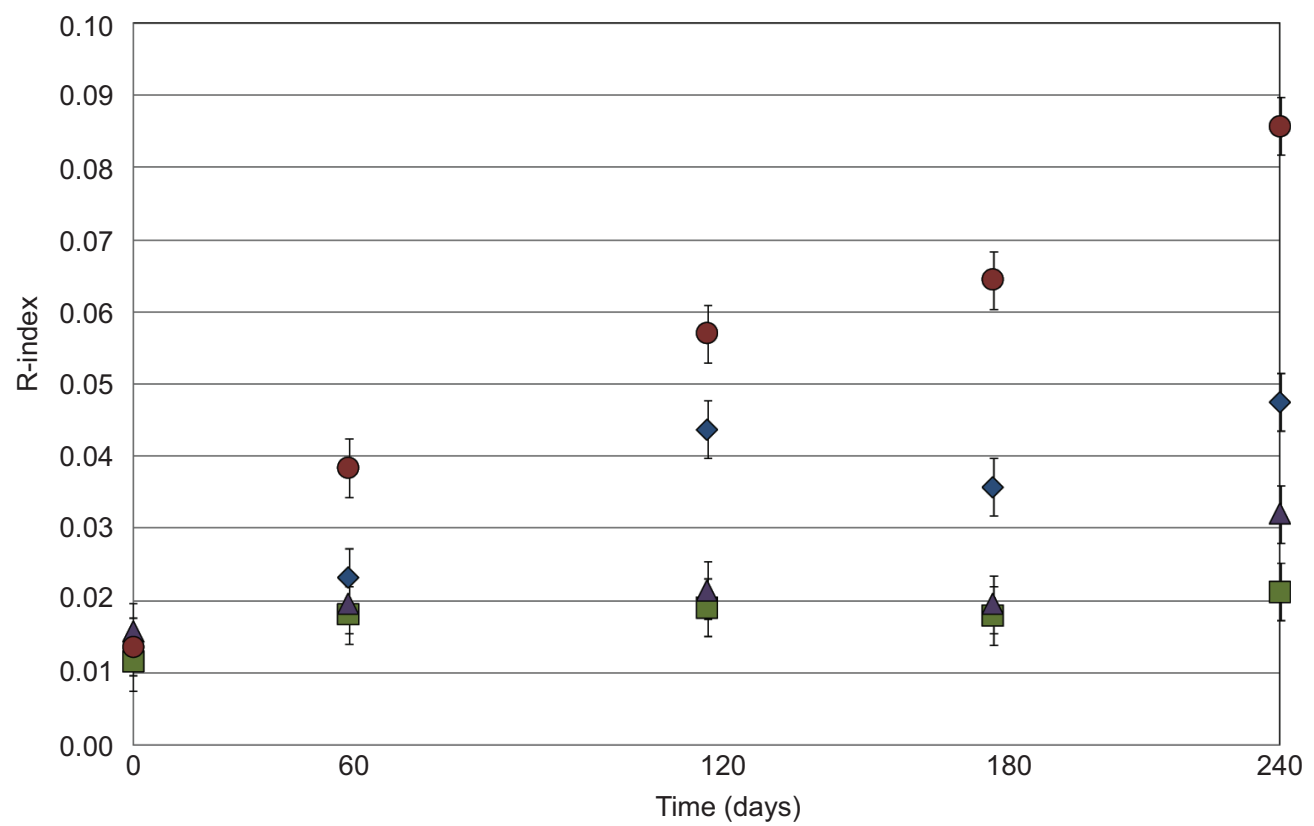

Figure 2. Mean value, standard error of R-index in samples of virgin oil (VO; red circle and line), olive oil containing water only (WO; blue diamond and line), olive oil containing solid particles (SO; purple triangle and line), and filtered oil (FO; green square and line) during storage. The $R^{2}$ and $A D J-R^{2}$ values of $R$-index were 0.8343 and 0.8157 , respectively.

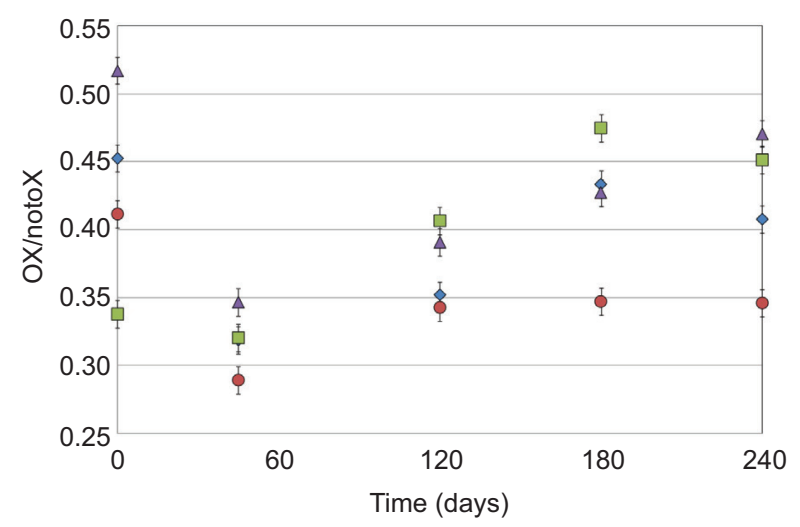

Figure 3. Mean value and standard error of phenolic oxidized-not oxidized form ratio (OX:not OX) in virgin oil vo (red circle), olive oil containing water only WO (blue diamond), olive oil containing solid particles SO (purple triangle), and filtered oil FO (green square) samples during storage. The $R^{2}$ and ADJ- $R^{2}$ of OX/not OX were 0.1957 and 0.1055 , respectively.

effect of treatment was determined in some single and some C5 and C6 LOX pathway volatile compounds, with lower content in SO samples than in FO, WO, and VO samples because of stripping caused by freeze-drying. No statistically significant differences during storage period and no significant interactions between filtration and storage period were determined in all the evaluated volatile compounds of LOX pathway and those related to 'fusty' defect.
The main effect of treatment and storage period and their interactions were not statistically significant for the unpleasant volatile compounds related to 'rancid' defect.

\section{Sensory evaluation}

The sensory attributes were evaluated and a significant $(p$ $\leq 0.05$ ) effect of treatment and storage period was determined. The positive 'fruity' attribute decreased during storage period in all samples. The $\mathrm{VO}$ and $\mathrm{SO}$ samples were significantly less fruity than FO and WO samples after 120 days of production (Table S1).

The negative 'fusty' and 'winey' defects, both related to microbial activity, and 'rancid' defect, related to oxidation, showed significant $(p \leq 0.001)$ increase during storage, and were of higher values in $\mathrm{VO}$ samples than in FO, SO, and WO samples after 45 days (Table S1). Furthermore, interactions between filtration and storage period were statistically significant for 'fusty', 'winey' and 'rancid' defects. Indeed, these defects increased faster in VO samples than in FO, WO, and SO samples (Figure 6).

The bitterness and pungency attributes significantly ( $p \leq$ 0.001 ) decreased in intensity during storage (Table S1). The VO samples were significantly $(p \leq 0.001)$ less bitter and pungent than $\mathrm{SO}$ and $\mathrm{FO}$ samples after 45 days. WO samples were not tasted due to filtration with glass wool. 

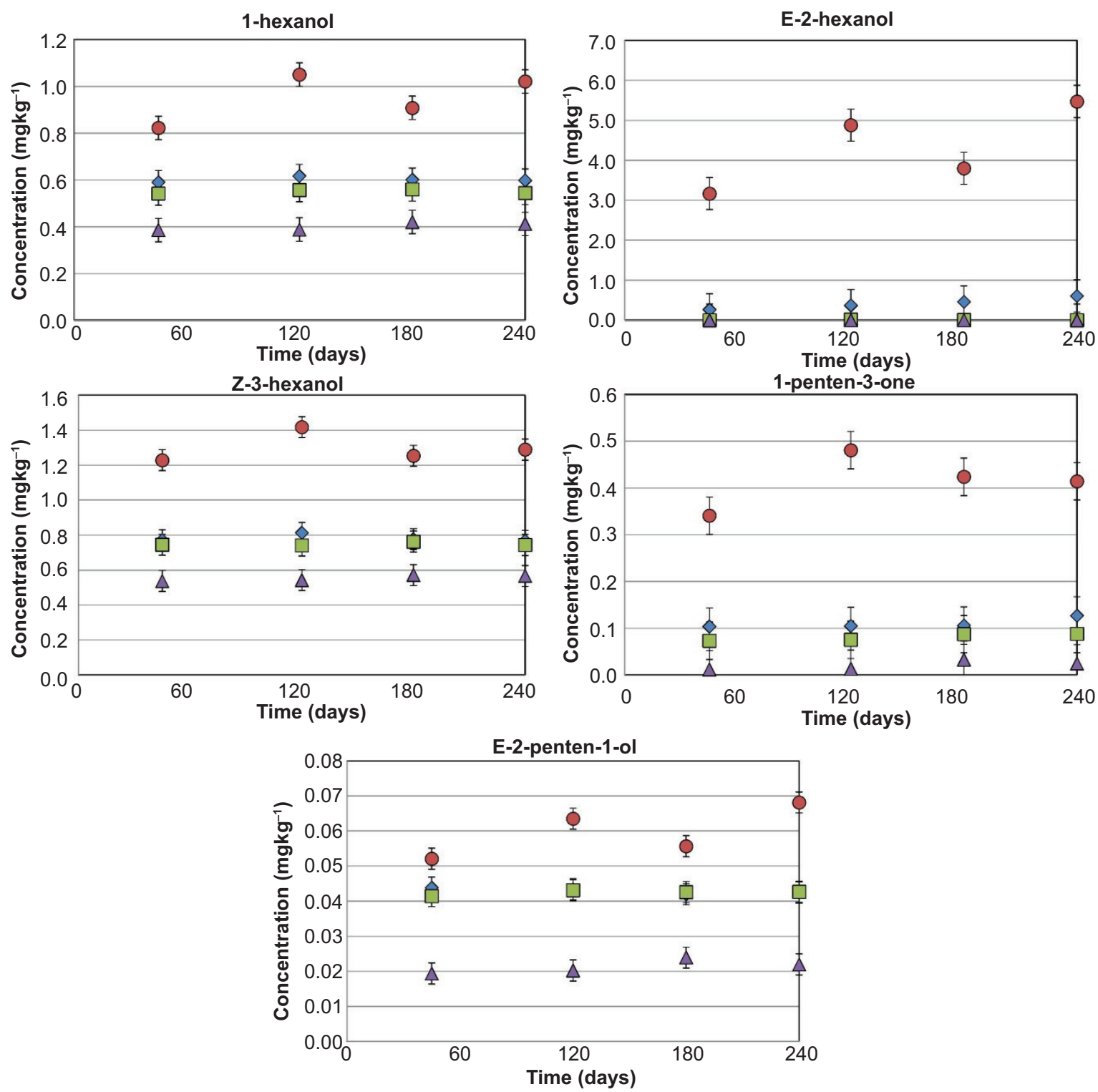

Figure 4. Mean contents and standard error of lipoxygenase (LOX) pathway volatile compounds in virgin oil VO (red circle), olive oil containing water only WO (blue diamond), olive oil containing solid particles SO (purple triangle), and filtered oil FO (green square) samples during storage. Only compounds statistically significant different $(p \leq 0.05)$ for time and/or treatment are reported. The $R^{2}$ and ADJ- $R^{2}$ values for LOX pathway volatile compound are as follows: 1 -hexanol, $R^{2}=0.5003, A D J-R^{2}=0.4442$; E-2-hexenol, $R^{2}=0.6473$, ADJ- $R^{2}=0.6077 ; Z$ Z-3-hexenol, $R^{2}=0.7068, A D J-R^{2}=0.6740 ; 1$-peten-3-one, $R^{2}=0.5996, A D J-R^{2}=0.5547$; and E-2-penten-1-ol, $R^{2}=0.7460, A D J-R^{2}=0.7175$.

\section{Discussion}

The experimental data highlighted that water and solid particles had some specific roles to play in the quality evolution of EVOO during storage. The obtained results demonstrated that two degradation phenomena, hydrolysis and microbial activity, were faster in $\mathrm{VO}$ samples than in FO, WO, and SO samples.

The presence of water micro-droplets dispersed in oil matrix increased the water/oil exchange surface, and the hydrolysis reaction occurred to a significant extent (XENAKIS et al., 2010). The enzymatic hydrolysis of triglycerides produced, not esterified fatty acids, that increased the FFA value more in VO samples than in FO, SO, and WO samples. Furthermore, the formation of phenolic compounds with low molecular weight, such as hydroxytyrosol and tyrosol (due to chemical hydrolysis of phenolic compounds (Zanoni, 2014; Cinquanta et al., 1997)), was higher in VO samples than in $\mathrm{FO}, \mathrm{SO}$, and $\mathrm{WO}$ samples. R-index confirmed the above trend in $\mathrm{VO}$ samples and established that WO 


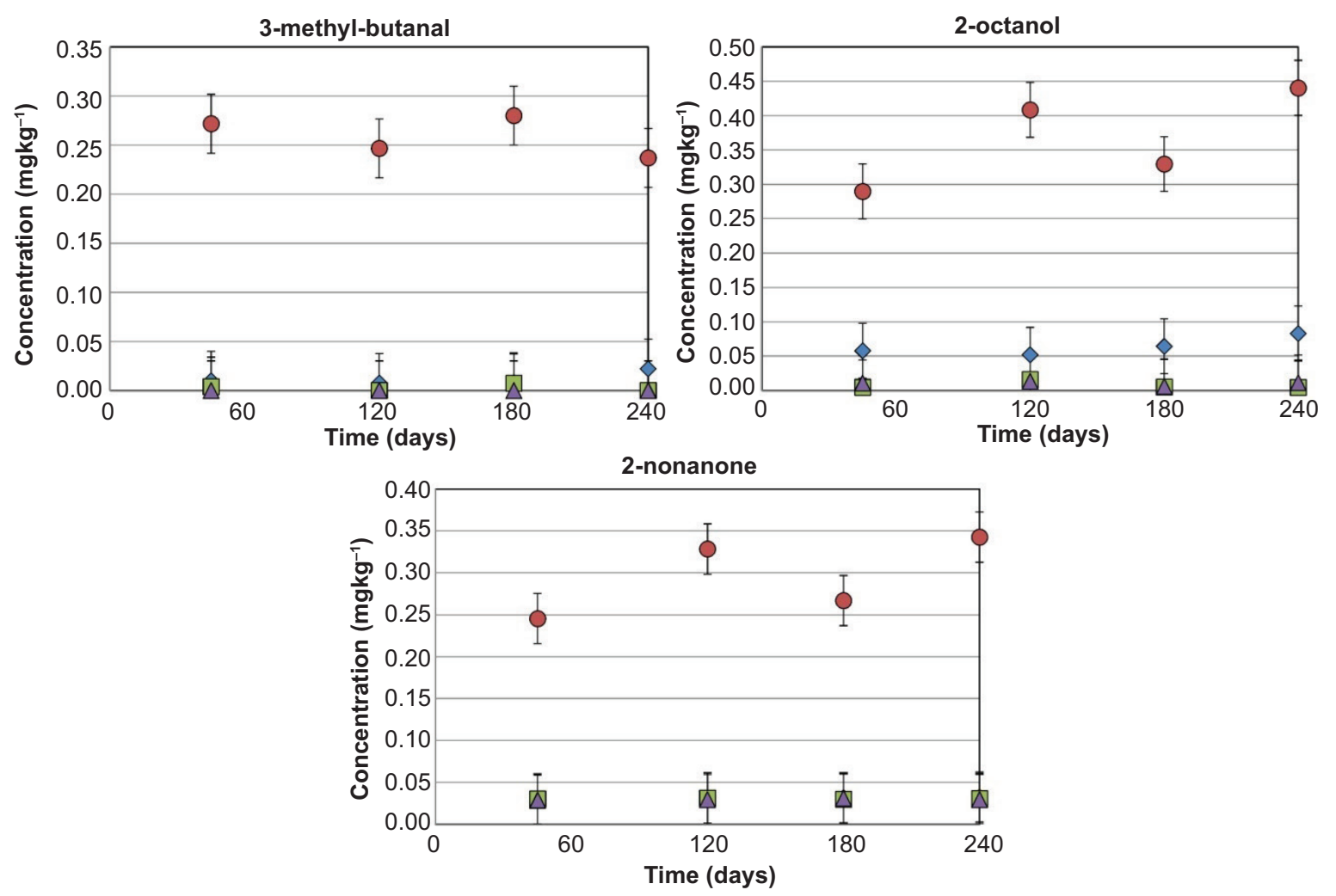

Figure 5. Mean contents and standard error of volatile compounds related to 'fusty' defect in virgin oil VO (red circle), olive oil containing water only WO (blue diamond), olive oil containing solid particles SO (purple triangle), and filtered oil FO (green square) samples during storage. Only compounds statistically significant different $(p \leq 0.05)$ for time and/or treatment are reported. The $R^{2}$ and $A D J-R^{2}$ values for 'fusty' defect volatile compounds are as follows: 3-methyl-butanal, $R^{2}=0.4201, A D J-R^{2}=$ $0.3551 ;$ 2-octanol, $R^{2}=0.7852, A D J-R^{2}=0.7611$; and 2-nonanone, $R^{2}=0.5197, A D J-R^{2}=0.4659$.

samples, with intermediate water content, had intermediate hydrolytic activity (Figure 2). The cause and effect relationship between the presence of micro-droplets of water in VO samples and the chemical hydrolytic phenomena of phenolic compounds were in accordance with the experimental data given in literature (Guerrini et al., 2020b).

The 'fusty' and 'winey' sensory defects and their related volatile compounds were strictly connected to the microbial activity. The microorganism cell count in VO samples was higher than in FO, SO, and WO samples during storage; the microbial survival was due to the favorable environment of VO samples, starting with water activity of $>0.6$ (Derossi et al., 2011), resulting in unpleasant volatile microbial metabolites, such as 3-methyl-butanal, 2-octanol, 2-nonanone (Figure 5).

The microbial activity was also helped by the content of solid particles. Our results highlight that water has to be combined with solid particles for microbial growth. WO and SO samples were not good for microbial survival, and only VO samples had favorable conditions for microbial growth (Figure 1).
The content of solid particles could be involved in promoting the transfer of phenols transfer from solid particles to oil. The SO samples were able to show the above effect, thanks to both absence of water and slow hydrolytic phenomena of phenolic compounds. The significant higher contents of both total phenolic compounds and sum of oleuropein and its derivatives in SO samples (Table 2) could be explained by the mass transfer of phenolic compounds from solid particles to oil. Solid particles consist of olive pulp and core fragments that are rich in high molecular weight phenolic compounds (Jerman Klen et al., 2015; Cecchi et al., 2018; Morales et al., 2005). However, the freeze-drying conditions led to initial oxidation, as shown in OX:not OX ratio values (Figure 3), and stripping of volatile compounds which affected quality parameters, such as K270 (Table 1), and development of 'rancid' defect (Figure 6).

Derived from the experimental results, following are the other functions of water and solid particles in the quality evolution of EVOO during storage, although they had some uncertain aspects.

The water content seemed to promote the LOX enzymatic pathway, which is responsible for 'fruity' positive 

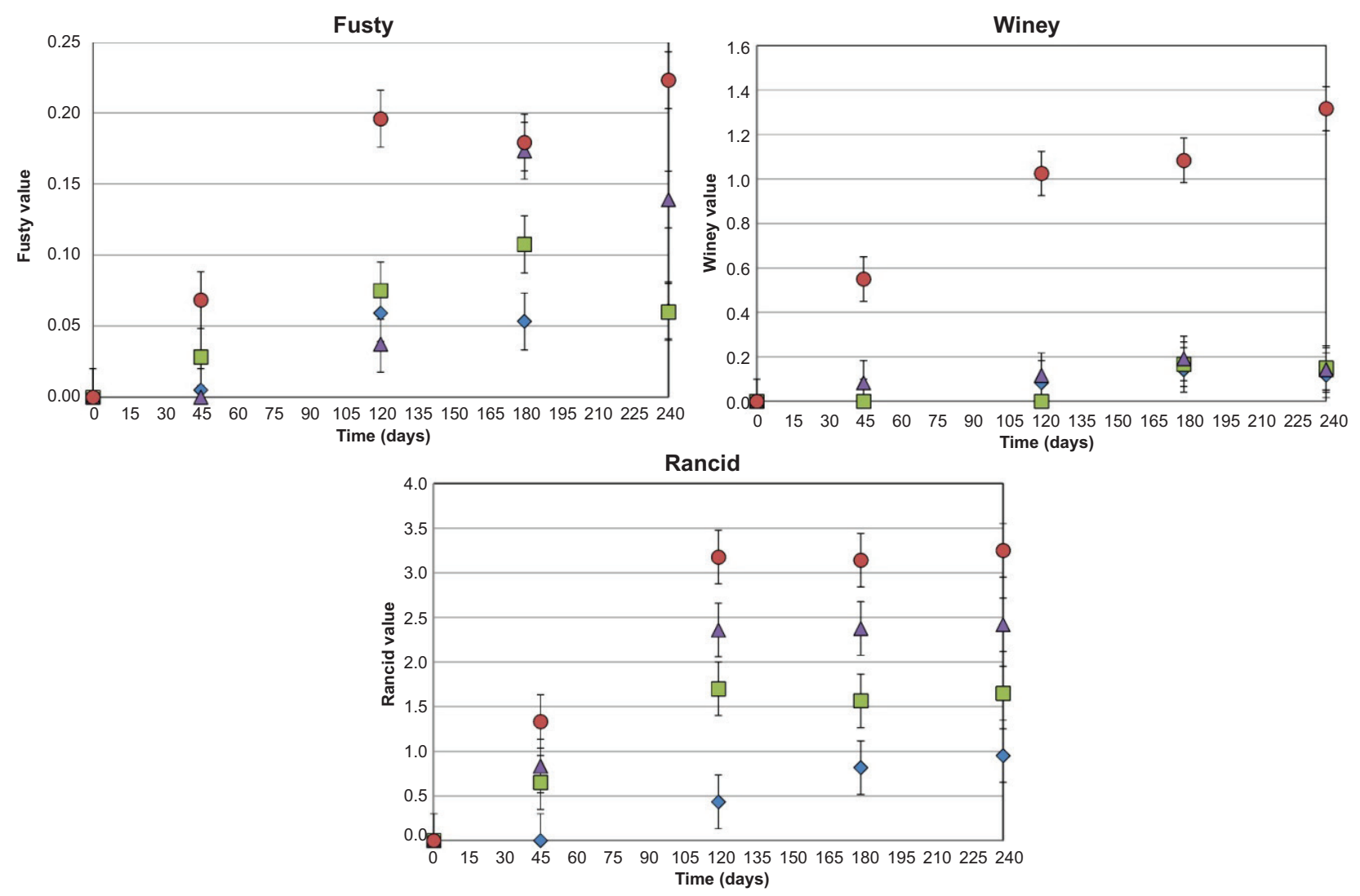

Figure 6. Mean contents and standard error of the 'fusty', 'winey', and 'rancid' defect scores in virgin oil Vo (red circle), olive oil containing water only WO (blue diamond), olive oil containing solid particles SO (purple triangle), and filtered oil FO (green square) samples during storage. The $R^{2}$ and $A D J-R^{2}$ for each sensory attribute are reported below: 'fusty', $R^{2}=0.4908, A D J-R^{2}=$ 0.4337 ; 'winey', $R^{2}=0.6216, A D J-R^{2}=0.5792 ;$ 'rancid', $R^{2}=0.5960, A D J-R^{2}=0.5507$.

sensory attributes. The content of $\mathrm{C} 5$ and $\mathrm{C} 6$ volatile compounds of LOX pathway was higher in VO samples than in FO and WO samples (Figure 4); however, VO samples had a significant low level of 'fruity' sensory attributes than determined in FO and WO samples. We suppose that significant appearance of 'fusty' defect led panelists to measure decrease in the 'fruity' score of VO samples (Guerrini et al., 2020a).

The water content also seemed to protect EVOO against negative oxidative phenomena during storage. The OX:not OX ratio of phenolic compounds (Figure 3) was higher in FO and SO samples than in WO and VO samples because of the stabilizing effect of water on oxidative degradation, as demonstrated in literature (Lercker et al., 1994; Ambrosone et al., 2002; Koidis and Boskou, 2006; Frega et al., 1999). However, the protective effect of water was not shown for chemical parameters, K232, K270, and $\Delta \mathrm{K}$, which did not increase significantly during storage as a function of treatments. The effect of treatments was not statistically significant for unpleasant volatile compounds, commonly related to 'rancid' sensory defect. Instead, the 'rancid' sensory defect behavior during storage demonstrated an opposite trend to the above oxidation phenomena: the 'rancid' scores were higher in VO samples than in FO, SO, and WO samples. The significant appearance of 'fusty' defect led panelists to measure an increase in the 'rancid' score of VO samples, since these two defects are characterized by some common volatile compounds (Morales et al., 2005).

\section{Conclusions}

In this study, an original approach was carried out to understand the significance of $\mathrm{VO}$ in terms of preservation of EVOO quality during storage. A clear effect of water content on hydrolytic phenomena and microbial activity was evidenced. Effect of content of solid particles to promote microbial activity was also demonstrated, potentially resulting in the loss of EVOO quality.

The results of the present study asserted that the recommended technique to avoid significant degradation during storage was to quickly filter freshly produced olive oil. However, an immediate filtration is not always possible as veiled olive oil is the product sought for bottling by producers. Therefore, A qualification of oil turbidity, 
based on separate measurement of water and insoluble solids contents, is suggested during different processing steps of olive oil chain, such as VO storage in mills, VO supply and storage in oil blenders, and transportation and distribution of veiled EVOO. It follows that, for olive oil producers, the qualification of veiled olive oil in potentially different combinations of water and solid contents (i.e., high-high, high-low, low-high, or low-low) could be useful to plan and control both water/solid separation techniques and storage of oil.

\section{Author Contributions}

Lorenzo Guerrini, Alessandro Parenti, and Bruno Zanoni did conceptualization; Carlotta Breschi and Lorenzo Guerrini curated the data. Formal analysis was done by Carlotta Breschi, Ferdinando Corti, and Luca Calamai. Funding acquisition was done by Alessandro Parenti and Bruno Zanoni. Methodology was done by Luca Calamai and Paola Domizio; and software handling was done by Carlotta Breschi and Lorenzo Guerrini. Supervision was carried out by Alessandro Parenti and Bruno Zanoni. Original draft was written by Carlotta Breschi and Lorenzo Guerrini; and the final writing-review and editing-was done by Lorenzo Guerrini, Alessandro Parenti, and Bruno Zanoni.

\section{Funding}

This study was supported by the AGER 2 Project, Grant No. 2016-0174, COMPETiTiVE-Claims of Oliveoil to IMProvE The marketValuE of the product.

\section{Conflict of Interest}

The authors declare no conflict of interest.

\section{References}

Ambrosone, L., Angelico, R., Cinelli, G., Di Lorenzo, V., Ceglie, A., et al. 2002. The role of water in the oxidation process of extra virgin olive oils. J. Am. Oil Chem. Soc. 79(6), 577-582. https:// doi.org/10.1007/s11746-002-0525-3

Bimbo, F., Roselli, L., Carlucci, D., and de Gennaro, B.C. 2020. Consumer misuse of country-of-origin label: insights from the Italian extra-virgin olive oil market. Nutrients. 12(2150), 1-12. https://doi.org/10.3390/nu12072150

Brenes, M., Garcia, A., Garcia, P., and Garrido, A. 2001. Acid hydrolysis of secoiridoida glycons during storage of virgin olive oil. J. Agr. Food Chem. 49(11), 5609-5614. https://doi.org/10.1021/ jf0107860

Breschi, C., Guerrini, L., Domizio, P., Ferraro, G., Calamai, L., Canuti, V., et al., 2019. Physical, chemical, and biological characterization of veiled extra virgin olive oil turbidity for degradation risk assessment. Eur. J. Lipid Sci. Tech. 121(11), 1900195, 1-6. https://doi.org/10.1002/ejlt.201900195

Bubola, K.B., Lukić, M., Mofardin, I., Butumović, A., and Koprivnjak, O. 2017. Filtered vs. naturally sedimented and decanted virgin olive oil during storage: effect on quality and composition. LWT. 84, 370-377. https://doi.org/10.1016/j.lwt.2017.05.069

Cayuela, J.A., Gómez-Coca, R.B., Moreda, W., and Pérez-Camino, M.D.C. 2015. Sensory defects of virgin olive oil from a microbiological perspective. Trends Food Sci. Tech. 43(2), 227-235. https://doi.org/10.1016/j.tifs.2015.02.007

Cayuela-Sánchez, J.A. and Caballero-Guerrero, B. 2019. Fresh extra-virgin olive oil, with or without veil. Trends Food Sci. Tech. 83, 78-85. https://doi.org/10.1016/j.tifs.2018.11.014.

Cecchi, L., Breschi, C., Migliorini, M., Canuti, V., Fia, G., Mulinacci, N., et al. 2019. Moisture in rehydrated olive paste affects oil extraction yield and phenolic compound content and profile of extracted olive oil. Eur. J. Lipid Sci. Tech. 121(4), 1800449, 1-10. https://doi.org/10.1002/ejlt.201800449

Cecchi, L., Migliorini, M., Zanoni, B., Breschi, C., and Mulinacci, N. 2018. An effective HPLC-based approach for the evaluation of the content of total phenolic compounds transferred from olives to virgin olive oil during the olive milling process. J. Sci. Food Agr. 98(10), 3636-3643. https://doi.org/10.1002/jsfa.8841

Ciafardini, G. and Zullo, B.A. 2002a Survival of micro-organisms in extra-virgin olive oil during storage. Food Microbiol. 19(1), 105-109. https://doi.org/10.1006/fmic.2001.0458

Ciafardini, G. and Zullo, B.A. 2002b. Microbiological activity in stored olive oil. Int. J. Food Microbiol. 75(1-2), 111-118. https:// doi.org/10.1016/S0168-1605(01)00739-5

Ciafardini, G. and Zullo, B.A. 2018. Virgin olive oil yeasts: a review. Food Microbiol. 70, 245-253. https://doi.org/10.1016/j. fm.2017.10.010

Cinquanta, L., Esti, M., and La Notte, E. 1997. Evolution of phenolic compounds in virgin olive oil during storage. J. Am. Oil Chem. Soc. 74(10), 1259-1264. https://doi.org/10.1007/ s11746-997-0054-8

Derossi, A., Severini, C., and Cassi, D. 2011. Mass transfer mechanisms during dehydration of vegetable food: traditional and innovative approaches. Ch. 15. In: "Advanced topics in mass transfer". IntechOpen (Ed.), pp. 305-354. https://doi.org/10.5772/14725

El haouhay, N., Samaniego-Sánchez, C., Asehraou, A., Jesús, R., Villalón-Mir, M., et al. 2018. Effects of olive storage and packaging on microbial and fatty acids profiles of olive oil produced in traditional mills in Morocco. J. Mat. Env. Sci. 2508, 854-863. https://doi.org/10.26872/jmes.2018.9.3.94

El Riachy, M., Priego-Capote, F., León, L., Rallo, L., and Luque de Castro, M.D. 2011. Hydrophilic antioxidants of virgin olive oil. Part 1: Hydrophilic phenols: a key factor for virgin olive oil quality. Eur. J. Lipid Sci. Tech. 113(6), 678-691. https://doi. org/10.1002/ejlt.201000400

European Union Commission. 2003. Commission implementing regulation (EC) No. 2016/2095 of 26 September 2016 amending regulation No. 2568/91 on the characteristics of olive oil and olive-residue oil and on the relevant methods of analysis. Off. J. Eur. Union. L 295, 57-77. 
Fiorini, D., Boarelli, M.C., Conti, P., Alfei, B., Caprioli, G., Ricciutelli, M., et al. 2018. Chemical and sensory differences between high price and low price extra-virgin olive oils. Food Res. Int. 105, 65-75. https://doi.org/10.1016/j.foodres.2017.11.005

Fortini, M., Migliorini, M., Cherubini, C., Cecchi, L., and Calamai, L. 2017. Multiple internal standard normalization for improving HS-SPME-GC-MS quantitation in virgin olive oil volatile organic compounds (VOO-VOCs) profile. Talanta. 165, 641652. https://doi.org/10.1016/j.talanta.2016.12.082

Fortini, M., Migliorini, M., Cherubini, C., Cecchi, L., Guerrini, L., Masella, P., et al. 2016. Shelf life and quality of olive oil filtered without vertical centrifugation. Eur. J. Lipid Sci. Tech. 118(8), 1213-1222. https://doi.org/10.1002/ejlt.201500229

Frega, N., Mozzon, M., and Lercker, G. 1999. Effects of free fatty acids on oxidative stability of vegetable oil. J. Am. Oil Chem. Soc. 76(3), 325-329. https://doi.org/10.1007/s1174.6-999-0239-4.

Fregapane, G., Lavelli, V., León, S., Kapuralin, J., and Desamparados Salvador, M. 2006. Effect of filtration on virgin olive oil stability during storage. Eur. J. Lipid Sci. Tech. 108(2), 134-142. https:// doi.org/10.1002/ejlt.200501175

Guerrini, L., Breschi, C., Zanoni, B., Calamai, L., Angeloni, G., Masella, P., et al. 2020a. Filtration scheduling: quality changes in freshly produced virgin olive oil. Foods. 9(8), 1067, 1-14. https:// doi.org/10.3390/foods9081067

Guerrini, L., Zanoni, B., Breschi, C., Angeloni, G., Masella, P., Calamai, L., et al. 2020b. Understanding olive oil stability using filtration and high hydrostatic pressure. Molecules. 25(2), 420, 1-15. https://doi.org/10.3390/molecules25020420

Guerrini, S., Mari, E., Migliorini, M., Cherubini, C., Trapani, S., Zanoni, B., et al. 2015. Investigation on microbiology of olive oil extraction process. Italian J. Food Sci. 27(2), 236-247. https:// doi.org/10.14674/1120-1770/ijfs.v190

International Olive Council (IOC). 2018. Best practice guidelines for the storage of olive oils and olive-pomace oils for human consumption. IOC/BPS/DOC.1/2018. International Olive Council, Madrid, Spain.

International Olive Council (IOC). 2017. Determination of biophenols in olive oils by HPLC. IOC/T.20/Doc. No. 29/Rev. 1/2017. International Olive Council, Madrid, Spain.

International Olive Council (IOC). 2018a. Guide for the selection, training and quality control of virgin olive oil tasters-qualifications of tasters, panel leaders and trainers. IOC/T.20/Doc. No. 14/Rev. 5/2018. International Olive Council, Madrid, Spain.

International Olive Council (IOC). 2018b Sensory analysis of olive oil. method for the organoleptic assessment of virgin olive oil. IOC/T.20/Doc. No. 15/Rev. 10/2018. International Olive Council, Madrid, Spain.

JermanKlen, T., GolcWondra, A., Vrhovšek, U., Sivilotti, P., and Vodopivec, B.M. 2015. Olive fruit phenols transfer, transformation, and partition trail during laboratory-scale olive oil processing. J. Agr. Food Chem. 63(18), 4570-4579. https://doi. org/10.1021/jf506353z

Koidis, A. and Boskou, D. 2006. The contents of proteins and phospholipids in cloudy (veiled) virgin olive oils. Eur. J. Lipid Sci. Tech. 108(4), 323-328. https://doi.org/10.1002/ejlt.200500319
Koidis, A., Triantafillou, E., and Boskou, D. 2008. Endogenous microflora in turbid virgin olive oils and the physicochemical characteristics of these oils. Eur. J. Lipid Sci. Tech. 110(2), 164171. https://doi.org/10.1002/ejlt.200700055

Lercker, G., Frega, N., Bocci, F., and Servidio, G. 1994. Veiled extra-virgin olive oils: dispersion response related to oil quality. J. Am. Oil Chem. Soc. 71(6), 657-658. https://doi.org/10.1007/BF02540597

Lozano-Sánchez, J., Cerretani, L., Bendini, A., Segura-Carretero, A., and Fernández-Gutiérrez, A. 2010. Filtration process of extra virgin olive oil: effect on minor components, oxidative stability and sensorial and physicochemical characteristics. Trends Food Sci. Tech. 21(4), 201-211. https://doi.org/10.1016/j. tifs.2009.12.004

Migliorini, M., Cherubini, C., Zanoni, B., Mugelli, M., Cini, E., and Berti, A. 2009. Influence of operating conditions of malaxation on the quality of extra virgin olive oil. Riv. It. Sostanze Grasse. 86(2), 92-102.

Morales, M.T., Luna, G., and Aparicio, R. 2005. Comparative study of virgin olive oil sensory defects. Food Chem. 91(2), 293-301. https://doi.org/10.1016/j.foodchem.2004.06.011

Romo-Sánchez, S., Alves-Baffi, M., Arévalo-Villena, M., ÚbedaIranzo, J., and Briones-Pérez, A. 2010. Yeast biodiversity from oleic ecosystems: study of their biotechnological properties. Food Microbiol. 27(4), 487-492. https://doi.org/10.1016/j.fm.2009.12.009

Xenakis, A., Papadimitriou, V., and Sotiroudis, T.G. 2010. Colloidal structures in natural oils. Curr. Opin. Colloid Interface Sci. 15(1-2), 55-60. https://doi.org/10.1016/j.cocis.2009.11.007

Zanoni, B. 2014. Which processing markers are recommended for measuring and monitoring the transformation pathways of main components of olive oil? Italian J. Food Sci. 26(1), 3-11.

Zullo, B.A., Cioccia, G., and Ciafardini, G. 2010. Distribution of dimorphic yeast species in commercial extra virgin olive oil. Food Microbiol. 27(8), 1035-1042. https://doi.org/10.1016/j. fm.2010.07.005

Zullo, B.A., Cioccia, G., and Ciafardini, G. 2013. Effects of some oilborn yeast on the sensory characteristics of Italian virgin olive oil during its storage. Food Microbiol. 36(1), 70-78. https://doi. org/10.1016/j.fm.2013.04.006

Zullo, B.A. and Ciafardini, G. 2018. Changes in physicochemical and microbiological parameters of short and long-lived veiled (cloudy) virgin olive oil upon storage in the dark. Eur. J. Lipid Sci. Tech. 120(1), 1700309, 1-8. https://doi.org/10.1002/ ejlt.201700309

Zullo, B.A., Pachioli, S., and Ciafardini, G. 2020. Reducing the bitter taste of virgin olive oil Don Carlo by microbial and vegetable enzymes linked to the colloidal fraction. Colloids Interfaces. 4(1), 11, 1-13. https://doi.org/10.3390/colloids4010011

Zullo, B.A. and Ciafardini, G. 2020a. Differential microbial composition of monovarietal and blended extra virgin olive oils determines oil quality during storage. Microorganisms. 8(3), 402, 1-16. https://doi.org/10.3390/microorganisms8030402

Zullo, B.A. and Ciafardini, G. 2020b. Virgin olive oil quality is affected by the microbiota that comprise the biotic fraction of the oil. Microorganisms. 8(5), 663, 1-13. https://doi. org/10.3390/microorganisms8050663 


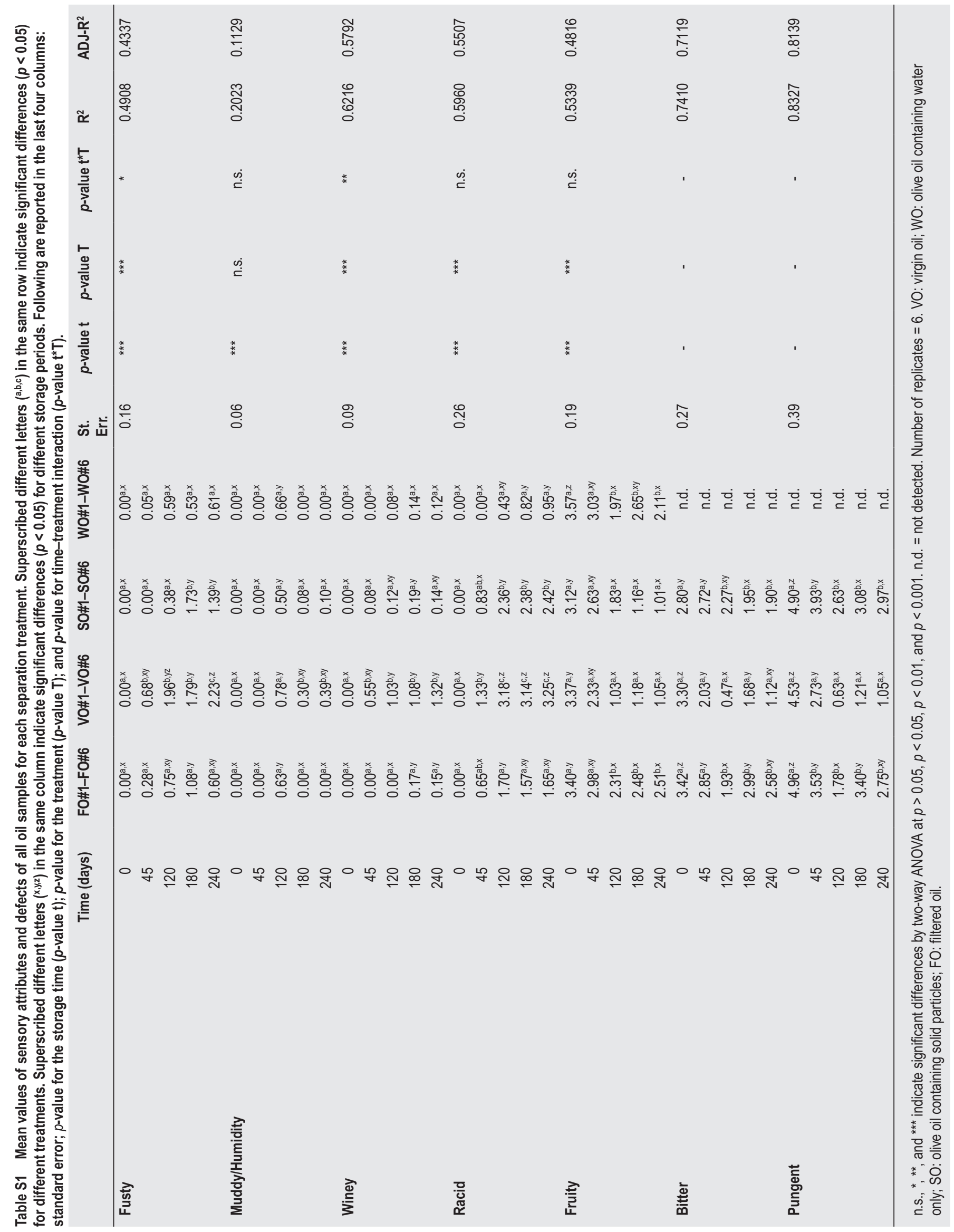

